\title{
A Novel Compressed Sensing-Based Approach for Fast MRI Reconstruction From Highly Under-Sampled K-Space Data
}

\author{
Chiara Di Martino ${ }^{1}$, Cristiana Corsi ${ }^{1}$, Damiana Lazzaro ${ }^{2}$ \\ ${ }^{1}$ DEI, University of Bologna, Cesena Campus, Cesena, Italy \\ ${ }^{2}$ DM, University of Bologna, Cesena Campus, Cesena, Italy
}

\begin{abstract}
Magnetic Resonance (MR) imaging is a multiparametric imaging technique allowing the diagnosis of a wide spectrum of cardiovascular diseases. Unfortunately, MR acquisitions tend to be slow, limiting patient throughput and limiting potential indications for use while driving up costs. Compressed sensing (CS) is a method for reducing MR scan time, increasing image reconstruction time. In this study we formulated a novel CS-based approach to speed up reconstruction procedure. A fidelity term that constrains the solution to be similar to the acquired samples was embedded in a nonconvex weighted total variation-based approach starting from highly subsampled $k$-space data. This approach was tested for the reconstruction of cardiac images in 10 delayed contrast enhanced MR (DCE-MR) acquisitions, using different $k$-space masks. Fully sampled MR images and the reconstructed images were compared by means of peakand signal-to-noise ratio (PSNR and SNR) metrics. Compared to other $k$-space filling trajectories, radial mask allowed the reconstruction of images of comparable quality (PSNR in [30 40]) but using less information. Overall, in all the test images we obtained a good reconstruction with similar SNR of the corresponding fully sampled images but using less than $20 \%$ of the original samples.
\end{abstract}

\section{Introduction}

Magnetic Resonance (MR) imaging is a multiparametric imaging technique allowing the diagnosis of a wide spectrum of cardiovascular diseases. This acquisition modality allows the investigation of a disease applying many study protocols for a comprehensive morphological and functional characterization of the cardiac disease. Unfortunately, cardiac MR imaging acquisitions tend to be slow, requiring in such cases, up to one hour for a single patient, in a very uncomfortable setting. In addition, a faster image acquisition might be used to shorten breath-hold times resulting in fewer motion-corrupted examinations.

Many algorithms were studied to speed up the MR acquisition procedure. Parallel imaging [1] is a widely used technique where the known placement and sensitivities of receiver coils are used to spatially localize the MR signal. The major drawback of such technique includes the reduction the signal-to-noise ratio, due to the decrease in the number of k-space lines, the geometric factor (which depends on the number, size and orientation of the different coil elements, resulting in a heterogeneous signal-to-noise ratio within the slice) and slice position, the acceleration factor and k-space filling trajectory, making MR images unsuitable for clinical use.

In the last ten years, a new approach was proposed named compressed sensing (CS). CS is a method for reducing MR scan time by acquiring less data through under-sampling of $\mathrm{k}$-space. Only a limited number of pixels/voxels in the $\mathrm{k}$-space is filled with data directly acquired and then, cardiac data are reconstructed. The philosophy of such approach was inspired by the research of Candes [2,3], Donoho [4], and Lustig [5] who stated: "Since the images we intend to acquire will be compressible, with most transform coefficients negligible or unimportant, is it really necessary to acquire all that data in the first place? Can we not simply measure the compressed information directly from a small number of measurements, and still reconstruct the same image which would arise from the fully sampled set? Furthermore, since MRI measures Fourier coefficients, and not pixels, wavelet, or DCT coefficients, the question is whether it is possible to do the above by measuring only a subset of $\mathrm{k}$ space."

Therefore, on one side, the availability of a CS approach, decreases acquisition time but prolongs image reconstruction time, since it requires execution of a computationally intensive optimization algorithm that iteratively estimates the whole image from the undersampled data.

The aim of this study was the design, implementation and test of a novel CS-based approach to speed up reconstruction procedure.

\section{Materials and Methods}

Compressed sensing is a signal processing technique for acquiring and reconstructing a signal, by finding solutions 


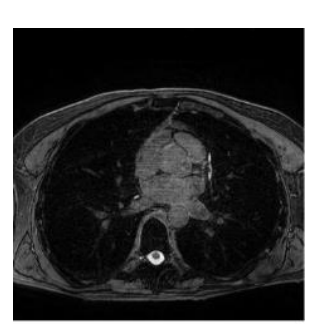

(a)
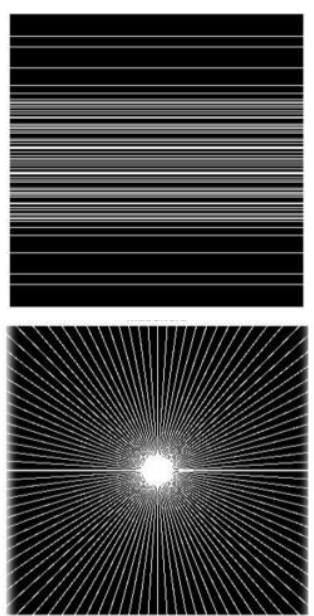

(b)
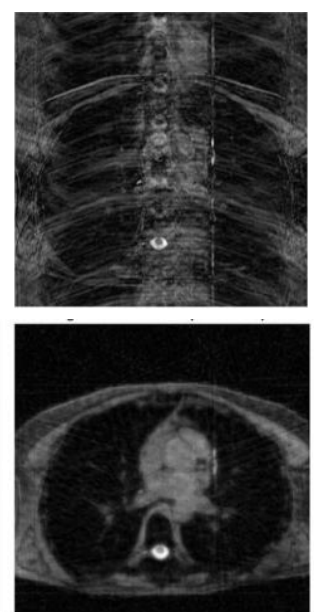

(c)

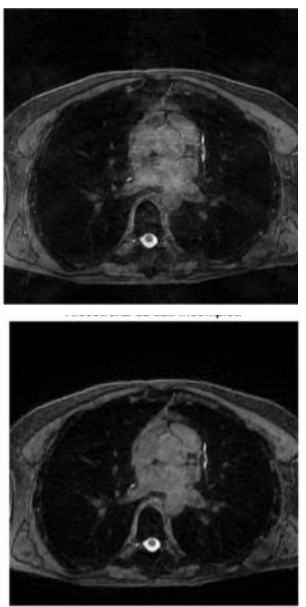

(d)

Fig. 1: (a) Original DCE MR image obtained with a fully sampled k-space; (b) cartesian (top) and radial (bottom) masks used to reconstruct the image; (c) final images reconstructed applying the inverse Fourier transform to the incomplete k-space; (d) final images reconstructed applying the proposed algorithm using 18\% (top) and 16\% (bottom) of the original image.

to underdetermined linear systems. This is based on the principle that, through optimization, the sparsity of a signal can be exploited to recover it from far fewer samples than required by the Nyquist-Shannon sampling theorem. The recovery process is possible only if two constraints are verified: (1) sparsity, which requires the signal to be sparse in some domain; (2) incoherence, which is applied through the isometric property, which is sufficient for sparse signals. MR signals satisfy both these constraints.

In particular, the MR images have a sparse gradient. The conventional CS framework for the reconstruction of a MR image starting from under-sampled data in k-space domain consists in solving an optimization problem, i.e. finding the image that minimizes this form:

$$
\min _{u} \frac{1}{2}\|\phi u-\mathrm{y}\|_{2}^{2}+\lambda F(\nabla u), \lambda>0
$$

where $\phi$ is the under-sampled $\mathrm{MxN}, \mathrm{M}<\mathrm{N}$, the Fourier transform matrix, obtained by pointwise multiplying the complete matrix Fourier transform for a binary mask $\mathrm{S}$, the $1_{2}$ term $\frac{1}{2}\|\phi u-\phi x\|_{2}^{2}$ is the fidelity-term and requires that the reconstruction $u$ is consistent in the Fourier domain with the acquired k-space data y, of $N$ components, $F$ is a function that induces sparsity on the gradient $\nabla u$ of the solution $\mathrm{u}, \nabla=\left[\begin{array}{l}\nabla_{x} \\ \nabla_{y}\end{array}\right]$, and $\lambda$ is the regularization parameter, balancing the fidelity term and the sparsity inducing function term.

One usual choice for the sparsity inducing function $F$ is the $1_{1}$-norm, namely $F(\cdot)=\|.\|_{1}$ and then

$$
F(\nabla u)=\sum_{i, j=1}^{N}\left|\nabla_{x} u_{i, j}\right|+\sum_{i, j=1}^{N}\left|\nabla_{y} u_{i, j}\right|
$$

that is the Total Variation of the image $u,(T V)$.

Recent results [6,7] have shown that the use of non- convex sparsity inducing function $\mathrm{F}(\cdot)$ allows to accurately and efficiently reconstruct a sparse signal from a lower number of measurements than those required in the case of the norm $l_{1}$ and seem to better reproduce the sparsing action of the $l_{0}$ quasi-norm compared to the $l_{1}$ norm. In this case, the minimization problem to be solve reads as:

$$
\min _{u} \frac{1}{2}\|\phi u-\phi x\|_{2}^{2}+\lambda \varphi(\nabla u), \lambda>0
$$

where $\varphi$ is a non-convex function depending by a $\mu$ parameter such that when $\mu \rightarrow 0$, the $\varphi$ function approaches to the $1_{0}$ quasi-norm. The resultant minimization problem is non-convex and for its solution we use an iterative reweighting scheme based on Forward-Backward splitting approach.

In this paper we propose, as preliminary study, to embed in the nonconvex weighted TV based approach a further fidelity term between the gradient of the solution and the gradient of an image $\mathrm{z}$ containing a good map of the gradient of the ideal image,

$$
\min _{u} \frac{1}{2}\|\phi u-\phi x\|_{2}^{2}+\gamma\|\nabla u-\nabla z\|_{2}^{2}+\lambda \varphi(\nabla u), \lambda, \gamma>0 .
$$

By integrating in the reconstruction process the fidelity to this gradient structure, we obtain the reconstruction quickly and more accurately respect than the conventional CS framework.

Our workflow requires the fully sampled cardiac MRI is represented in the $\mathrm{k}$-space. A mask is applied to select only few frequencies; the modified CS approach is then applied to this under-sampled data to reconstruct the image. 

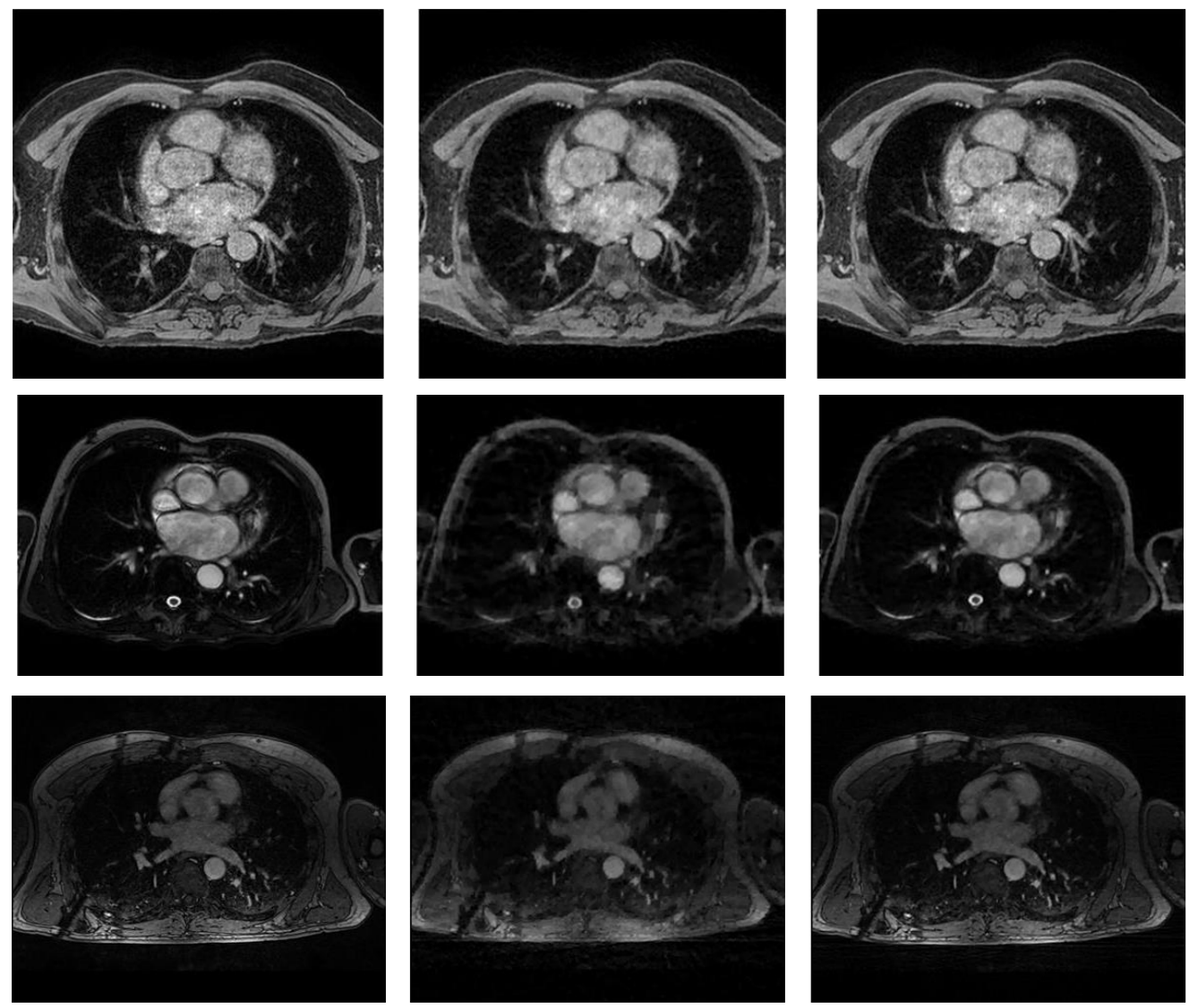

(a)

(b)

(c)

Fig. 2: (a) Fully sampled DCE MR images (top: $S N R=0.28$, mid: $S N R=0.26$, bottom: $S N R=1.5$ ); (b) reconstructed images with $P S N R=29$ (top: $S_{p}=17 \%$, \# of radial lines 53, $S N R=0.31$; mid: $S_{p}=7 \%$, \# of radial lines 29, $S N R=0.26$, bottom: $S_{p}=8 \%$, \# of radial lines 29, $S N R=1.3$ ); (c) reconstructed images with $P S N R=34$ (top: $S_{p}=29 \%$, \# of radial lines $95, S N R=0.35$; mid: $S_{p}=13 \%$, \# of radial lines 34, $S N R=0.29$, bottom: $S_{p}=23 \%$, \# of radial lines $95, S N R=1.44$ ).

We tested two masks: the cartesian and the radial mask. Each type was tested applying a different number of subsampling lines, 20 and 40 radial lines, 40 and 60 horizontal lines for the radial and cartesian masks, respectively.

This approach was tested for the reconstruction of cardiac images in 10 delayed contrast enhanced MR (DCEMR) acquisitions, using different k-space masks.

Fully sampled MR images and the reconstructed images obtained using a different number of sample lines were compared by means of peak- and signal-to-noise ratio (PSNR and SNR) metrics:

$$
\begin{gathered}
\text { PSNR }=20 \log _{10}\left(\frac{1}{r m s e}\right) \\
\text { rmse }=\sqrt{\frac{\sum_{i, j=1}^{N}\left(u_{i j}-x_{i j}\right)^{2}}{N^{2}}}
\end{gathered}
$$

where rmse is the root mean square error, $u$ is the reconstructed image and $x$ the original one; $N^{2}$ is the number of pixels in the image;

$$
S N R=\frac{\mu}{\sigma}
$$

where $\mu$ is the mean and $\sigma$ is the standard deviation of pixels in the image.

$P S N R$ values in the range $30 \div 40$ are associated with good image quality.

The percentage of information from $\mathrm{k}$-space used to reconstruct the image $S_{p}$ applying a mask $S$ was defined as:

$$
S_{p}=\frac{n n z(S)}{N^{2}}
$$

where $n n z(S)$ is the number of non-zero pixels in S.

\section{Results}

The algorithm was first tested to understand which mask allows the best reconstruction in terms of PSNR. An example is shown in Figure 1. Table 1 reports the results in five MR images.

Radial mask allowed the reconstruction of images of comparable quality but using less information compared to other k-space filling trajectories.

Additional tests were than performed considering the radial mask and the number of radial lines was chosen to optimize image quality. In all the 10 DCE-MR images we 

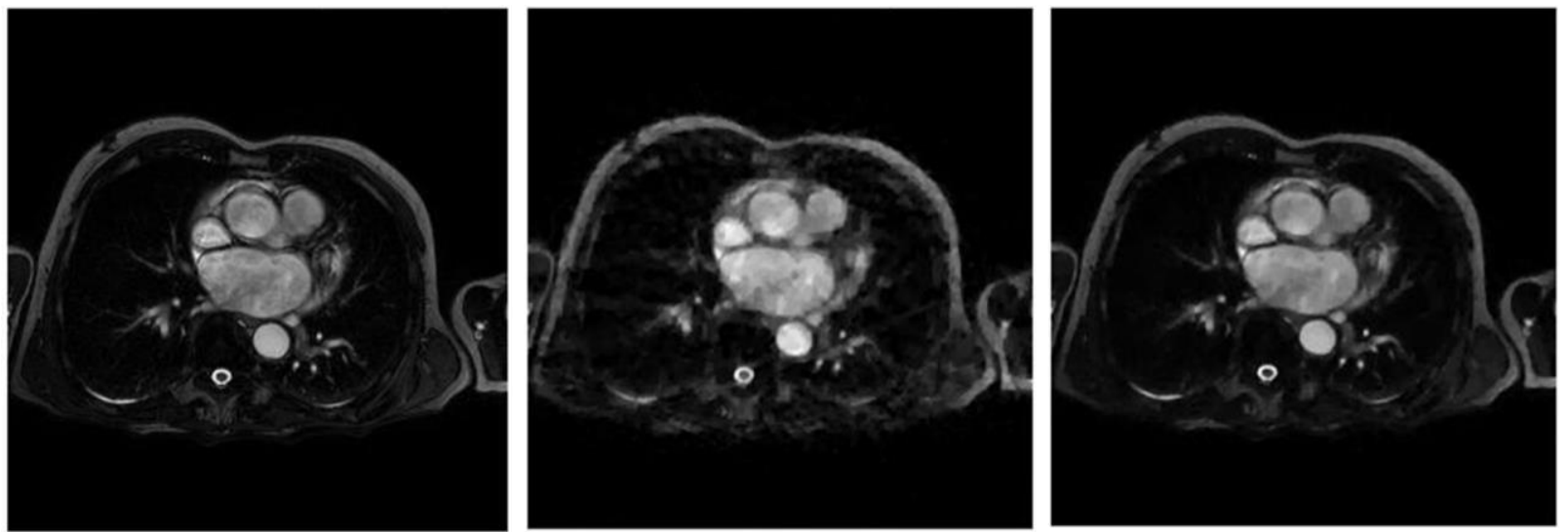

Fig. 3: Left: Original DCE MR image obtained with a fully sampled k-space; mid: reconstruction obtained applying the algorithm based on the fidelity and sparsity terms using 20 lines $\left(P S N R=30.5, S_{p}=8 \%\right)$; right: reconstructed image obtained using the same mask and adding the similarity term $\left(P S N R=35.3, S_{p}=8 \%\right)$.

Table 1. Results obtained applying our reconstruction algorithm to 5 sample images applying different masks, compared to direct reconstruction from incomplete $\mathrm{k}$-space

\begin{tabular}{|c|c|c|c|c|c|}
\hline \multirow{2}{*}{ Image 1 } & mask & $\begin{array}{c}\text { \# of } \\
\text { lines }\end{array}$ & $\begin{array}{c}\text { PSNR } \\
\text { incomplete } \\
\text { k-space }\end{array}$ & $\begin{array}{c}\text { PSNR } \\
\text { applying our } \\
\text { algorithm }\end{array}$ & $\begin{array}{c}\boldsymbol{S}_{\boldsymbol{p}} \\
(\%)\end{array}$ \\
\cline { 2 - 6 } & cartesian & 160 & 25 & 32.3 & 25 \\
\hline \multirow{2}{*}{ Image 2 } & radial & 80 & 33.6 & 37.7 & 13.8 \\
\cline { 2 - 6 } & cartesian & 60 & 18.7 & 27.9 & 18.1 \\
\hline \multirow{2}{*}{ Image 3 } & radial & 50 & 27.7 & 31.3 & 16.3 \\
\cline { 2 - 6 } & radial & 50 & 16.7 & 28.7 & 19.9 \\
\hline \multirow{2}{*}{ Image 4 } & cartesian & 70 & 26.3 & 41.2 & 27.3 \\
\cline { 2 - 6 } & radial & 40 & 32.2 & 39.5 & 17.5 \\
\hline \multirow{2}{*}{ Image 5 } & cartesian & 70 & 19.5 & 26.9 & 18.5 \\
\cline { 2 - 6 } & radial & 70 & 28.5 & 31.8 & 19.6 \\
\hline
\end{tabular}

obtained a good reconstruction with similar SNR of corresponding fully sampled images using less than $20 \%$ of the original samples. Few additional examples are shown in Figure 2.

By adding the similarity term based on the gradient of the image that may be derived by probability maps or atlas, quality results further improved and PNSR values of 35 were obtained using less than $10 \%$ of the original image (Figure 3).

\section{Discussion and Conclusion}

The proposed approach allowed a fast and accurate reconstruction compared to the conventional CS framework.

We obtained DCE MR images of quality comparable with the original images but using only a small percentage of the information of the k-space domain (about 20\%). Indeed, for all the MR images on which the algorithm was tested, it turned out that considering a value of PSNR approximately equal to 35 , with a number of radial lines different from each image test, our algorithm allows the reconstruction of images with $S N R$ very close to the SNR value of the image obtained by acquiring the complete kspace. The availability of such algorithm on board on a MR scanner would allow the acquisition of MR images of comparable quality but significantly decreasing the acquisition time and consequently patient's discomfort.

\section{References}

[1] A. Deshmane, V. Gulani, M.A. Griswold, N.E. Seiberlich, Parallel MR imaging. Magn Res Imaging. 2012;36(1):5572.

[2] E.J. Candes, J. Romberg and T. Tao. Robust uncertainty principles: exact signal reconstruction from highly incomplete frequency information. IEEE Trans Inf Theory 2006;52:489-509.

[3] E.J. Candès and T. Tao. Near optimal signal recovery from random pro-jections: Universal encoding strategies, IEEE Trans on Inform Theory 2006.

[4] D.L. Donoho. Compressed sensing. IEEE Trans Inf Theory 2006;52:1289-306.

[5] M. Lustig, D. Donoho, and J.M. Pauly. Sparse MRI: The Application of Compressed Sensing for Rapid MR Imaging. Magnetic Resonance in Medicine 2007;58:1182 1195.

[6] E.J. Candes, M.B. Wakin, S.P. Boyd, Enhancing sparsity by reweighted 11, Minimization Journal of Fourier Analysis and Applications 2008;14:877-905.

[7] L.B. Montefusco, D. Lazzaro, and S. Papi, A Fast Algorithm for Nonconvex Approaches to Sparse Recovery Problems, Signal Processing 2013;93:2636-2647.

Address for correspondence:

Chiara Di Martino

DEI, University of Bologna

Via dell'Università 50, 47521 Cesena, Italy

E-mail: chiara.dimartino@studio.unibo.it 